\title{
Noninvasive Detection of Changes in Cerebral Blood Flow by Near-Infrared Spectroscopy in a Piglet Model of Hydrocephalus
}

\author{
JANET S. SOUL, GEORGE A. TAYLOR, DAVID WYPIJ, ADRÉ J. DUPLESSIS, AND \\ JOSEPH J. VOLPE \\ Departments of Neurology [J.S.S., A.J.D., J.J.V.] and Radiology [G.A.T.], Children's Hospital and \\ Harvard Medical School, and Department of Biostatistics [D.W.], Harvard School of Public Health, \\ Boston, Massachusetts 02115, U.S.A.
}

\begin{abstract}
ABST
Formulation of rational interventions in infantile hydroceph-
alus is limited by the inability to monitor cerebral hemodynamics
quantitatively, continuously, and noninvasively. Near-infrared
spectroscopy (NIRS) measures changes in cerebral concentration
of oxygenated and deoxygenated hemoglobin (HbO $\mathrm{Hb}_{2}$ and Hb);
HbD is the derived difference between $\mathrm{HbO}_{2}$ and Hb. Our
previous work showed that HbD reflected cerebral blood flow
(CBF) measured by radioactive microspheres in a piglet model of
systemic hypotension. This study was designed to determine
whether NIRS detected important changes in cerebral perfusion
and oxygenation in a piglet model of hydrocephalus and whether
changes in HbD accurately reflected changes in CBF. Acute
hydrocephalus was produced in neonatal piglets by intraventric-
ular infusion of "mock cerebrospinal fluid." Intracranial pressure
(ICP) was maintained for several minutes at approximately 10,
20, and 30 mm Hg above the baseline ICP. CBF was measured
in cerebral cortex, white matter, and basal ganglia at each ICP by
radioactive microspheres. Changes in HbO ${ }_{2}$ and Hb were mea-
sured continuously by NIRS. Cerebral perfusion pressure de-
clined with increasing ICP, and this decline was accompanied by
significant decreases in HbD measured by NIRS and CBF mea-
sured by radioactive microspheres. There was a strong correla-
\end{abstract}
Rational management of infantile hydrocephalus requires information about the disturbances in cerebral perfusion and metabolism that may contribute to the development of brain injury. Substantial evidence indicates that impairments of cerebral perfusion and metabolism play a role in the pathogenesis of brain injury secondary to hydrocephalus (1-8). However, currently there are major limitations in the ability to define changes in cerebral hemodynamics and metabolism quantitatively, continuously, and noninvasively at the bedside of the human infant.

Received February 14, 2000; accepted April 18, 2000.

Correspondence and reprint requests: Joseph J. Volpe, M.D., Children's Hospital, Dept. of Neurology, Fegan 11, 300 Longwood Avenue, Boston, MA 02115, U.S.A.

Supported by grants from the Hearst Foundation (J.S.S.) and the National Institutes of Health (P30-HD-18655, J.J.V.). tion between changes in $\mathrm{HbD}$ and individual changes in $\mathrm{CBF}$ in cerebral cortex, white matter, and basal ganglia (all $p<0.0001$ ). This study demonstrates that changes in $\mathrm{HbD}$ reflect changes in CBF over a wide range of ICP in a model of acute hydrocephalus. This reproducible and easily obtained measurement by NIRS could facilitate considerably decisions concerning therapeutic interventions. (Pediatr Res 48: 445-449, 2000)
NIRS, near-infrared spectroscopy
Abbreviations
$\mathbf{H b O}_{2}$, oxygenated $\mathrm{Hb}$
$\mathbf{H b}$, deoxygenated $\mathrm{Hb}$
HbD, $\mathrm{Hb}$ difference signal
HbT, total $\mathrm{Hb}$
CytOx, oxidized cytochrome $\mathrm{aa}_{3}$
ICP, intracranial pressure
MAP, mean arterial pressure
CPP, cerebral perfusion pressure
CBF, cerebral blood flow
rCBF, regional cerebral blood flow
dpf, differential path-length factor

Doppler ultrasound provides information only about CBF velocity and cerebrovascular resistance but is not a consistently reliable measure of CBF (9). Positron emission tomography, magnetic resonance imaging, and magnetic resonance spectroscopy can supply detailed regional information regarding cerebral perfusion and metabolism but provide data at only a single point in time and require the transport of the critically ill infant to the scanning device.

NIRS is a relatively new technique with the capacity to detect changes in cerebral hemodynamics and oxygenation continuously and noninvasively at the bedside of the infant (10). NIRS is based on the principle that hemoglobin and CytOx (the terminal enzyme in the mitochondrial respiratory chain) are naturally occurring chromophores that absorb near-infrared light. The absorption 
characteristics of $\mathrm{Hb}$ and CytOx change as a function of their oxygenation state. NIRS quantitatively measures changes in $\mathrm{HbO}_{2}$ and $\mathrm{Hb}$ and thereby provides information about intravascular oxygenation. Changes in the redox state of CytOx reflect alterations in intracellular oxygenation. $\mathrm{HbT}$ is derived from $\mathrm{HbO}_{2}$ plus $\mathrm{Hb}$ and provides a measure of cerebral blood volume. $\mathrm{The} \mathrm{HbD}$, derived from $\mathrm{HbO}_{2}$ minus $\mathrm{Hb}$, should relate to cerebral oxygen delivery and, thereby, cerebral perfusion. The hypothesis that changes in $\mathrm{HbD}$ reflect changes in cerebral perfusion was addressed in a piglet model (11). In support of this notion, we showed in that model that changes in cerebral perfusion produced by hypotension resulted in changes in $\mathrm{HbD}$ that did reflect alterations in CBF measured simultaneously by radioactive microspheres, the current gold standard technique for determining $\mathrm{CBF}$ (11). Thus, we undertook this study to determine in a piglet model of acute hydrocephalus in which cerebral perfusion is altered by an increase in ICP 1 ) whether changes in cerebral perfusion and oxygenation accompanying acute hydrocephalus could be detected by NIRS, and 2) whether changes in $\mathrm{HbD}$ accurately reflected changes in CBF (as measured by radioactive microspheres) under these conditions.

\section{METHODS}

Surgical preparation. Seven newborn Yorkshire piglets (2-7 d old) were anesthetized with $20 \mathrm{mg} / \mathrm{kg}$ of ketamine hydrochloride (Ketalar; Parke-Davis, Morris Plains, NJ, U.S.A.) and $5 \mathrm{mg} / \mathrm{kg}$ of xylazine (Rompun; Miles, Shawnee, KA, U.S.A.) by intramuscular injection, followed by $1 \%$ halothane (Fluothane; Wyeth-Ayerst, Philadelphia, PA, U.S.A.) inhalation anesthesia. Thereafter, a continuous i.v. infusion of diprivan 1\% (Propofol; Stuart Pharmaceuticals, Wilmington, DE, U.S.A.) diluted in $5 \%$ dextrose at a dose of 0.02 $\mathrm{mg} \cdot \mathrm{kg}^{-1} \cdot \mathrm{min}^{-1}$ was used to maintain anesthesia for the remainder of the experiment. A heating lamp and warming blanket were used to prevent loss of body heat during anesthesia, and body temperature was monitored with a rectal probe.

Controlled ventilation was maintained via oral endotracheal tube, and supplemental oxygen was administered as needed to maintain oxygen saturation $>94 \%$. Oxygen saturation was monitored with a transcutaneous sensor placed on the left forelimb. An end-tidal carbon dioxide monitor was placed in line in the endotracheal tube, and ventilation was adjusted to maintain $\mathrm{PCO}_{2}$ at $35-40 \mathrm{~mm} \mathrm{Hg}$. A polyvinyl chloride catheter was introduced into the right femoral artery and the catheter threaded up the aorta until the tip was within the left cardiac ventricle by use of fluoroscopy to guide placement. The location of the catheter tip was confirmed throughout the experiment by the typical waveform. Catheters were also placed in the left femoral artery for continuous blood pressure monitoring and in both femoral veins for administration of i.v. fluid and medications. Arterial blood pressure and heart rate were monitored continuously with a Hewlett Packard model 76 physiologic monitoring system (Hewlett Packard, Andover, MA, U.S.A.). A $1 \times 2$-cm craniotomy was performed across the midline at the level of the coronal suture, leaving the dura intact for sonographic access to the brain [to guide placement of the catheter and to monitor ventricular enlargement with infusion of mock cerebrospinal fluid (CSF)]. ICP was monitored continuously with a probe placed in the epidural space (Steritek J7000 Intracranial Pressure Monitor). A 2-mm burr hole was created in the right parieto-occipital area, and a 24-gauge catheter was placed into the right lateral ventricle under sonographic guidance. Infusion of mock CSF into the ventricular system via this catheter produced the acute hydrocephalus, as outlined below. A $7.0-\mathrm{MHz}$ vector transducer (ACUSON 128 XP; Mountainview, CA, U.S.A.) was used for guidance of catheter placement and confirmation of resultant hydrocephalus.

Production of acute hydrocephalus. Mock CSF (dextrose $5 \%$ in one-fourth normal saline warmed to body temperature) was infused via the catheter into the right lateral ventricle to produce progressive ventriculomegaly and increases in ICP. The continuous infusion was adjusted to increase the ICP to approximately 10, 20, and $30 \mathrm{~mm} \mathrm{Hg}$ above the baseline ICP. After the ICP had been stabilized at each subsequent pressure for several minutes, radioactively labeled microspheres were injected into the left (cardiac) ventricle for measurement of $\mathrm{CBF}$, as outlined below. CPP was derived from the difference between MAP and ICP, which were both measured continuously throughout the experiment. Experiments were usually completed in approximately $45 \mathrm{~min}$ (range, 35-90 $\mathrm{min}$ ).

Measurement of $\mathbf{C B F}$ by microspheres. $\mathrm{rCBF}$ was determined by a radiolabeled microsphere technique. We used $16 \pm$ 0.5-mm microspheres labeled with four separate isotopes: 141Ce, 113-Sn, 103-Ru, and 46-Sc (Dupont-New England Nuclear Products, Boston, MA, U.S.A.). Independent measurement of each isotope is possible because each isotope has a different photon energy. For each blood flow determination, a volume containing $1.5 \times 10^{6}$ microspheres labeled with one of the above isotopes was injected into the left ventricle and flushed with $5 \mathrm{~mL}$ of saline over a period of $20 \mathrm{~s}$. A reference blood sample was withdrawn simultaneously from a femoral catheter at the time of each microsphere injection. At the end of each experiment, the animals were euthanized with an i.v. injection $(1 \mathrm{~mL} / 10 \mathrm{lb})$ of Fatalplus (Vortech Pharmaceuticals; Dearborn, MI, U.S.A.), and the brains were harvested. After sectioning, tissue samples of cortical gray matter, periventricular white matter, and basal ganglia from each hemisphere were dissected, weighed, and placed in $15-\mathrm{mL}$ vials for analysis in a gamma scintillation spectrometer (Cobra II, Gamma Counter; Packard Instruments, Meriden, CT, U.S.A.). Blood flow was determined by comparing the radioactivity for each radiolabel in each tissue sample and by comparing tissue radioactivity to the radioactivity in the reference blood samples. CBF was expressed in $\mathrm{mL} / 100 \mathrm{~g}$ tissue/min.

NIRS measurements. NIRS measurements were collected using a four-wavelength $(775,824,843$, and $909 \mathrm{~nm})$ spectrophotometer (Hamamatsu Photonics, Japan). The instrument transmits light generated by laser diodes via a fiber-optic bundle to an optode placed on the piglet's head. An identical fiber-optic bundle transmits the light received by a second optode to the photomultiplier tube where the photons are counted. NIRS optodes were placed on the skin overlying each cerebral hemisphere just anterior to the coronal suture with an 
interoptode spacing of $3.0 \mathrm{~cm}$. The optodes were covered with opaque material and secured to the head with adhesive tape.

Changes in the concentration of light attenuation at each wavelength were used to calculate changes in the relative concentration of each chromophore $\left(\mathrm{Hb}, \mathrm{HbO}_{2}\right.$, and CytOx) according to the Beer-Lambert law $(12,13)$. The calculations were corrected for the interoptode distance of $3.0 \mathrm{~cm}$. The dpf corrects the total path length of the light for the degree of scattering within the tissue sample. Because the dpf is not known for the newborn piglet brain, the calculations were not corrected by the dpf. Thus, NIRS measurements were expressed in units of $\mu \mathrm{mol} / \mathrm{L} \times \mathrm{dpf}$.

The NIRS instrument simultaneously recorded MAP, ICP, peripheral oxygen saturation, and end-tidal $\mathrm{PCO}_{2}$ along with the NIRS signals noted above. $\mathrm{HbT}$ and $\mathrm{HbD}$ were calculated from the sum and difference, respectively, of $\mathrm{HbO}_{2}$ and $\mathrm{Hb}$.

Data analysis. Linear regression methods were used to analyze the relationships between NIRS signals, rCBF measured by microspheres, and CPP. Generalized estimating equations were used to adjust $p$ values, taking into account repeated measures on subjects. Statistical analyses were performed with SAS software (SAS Institute, Inc., Cary, NC, U.S.A.). In one instance (see "Results"), a nonlinear mixed effects model was used, as the nonlinear logistic model showed a statistically better fit than the linear mixed model.

The present study was approved by our institutional Animal Care and Use Committee and complied with the guidelines of the National Institutes of Health for the care and handling of animals.

\section{RESULTS}

Seven piglets were studied with simultaneous measurements by NIRS and radioactive microspheres over a wide range of ICP and, thus, CPP. A data sample of continuously obtained NIRS signals ( $\mathrm{Hb}, \mathrm{HbO}_{2}$, and $\left.\mathrm{HbD}\right), \mathrm{ICP}$, and MAP from one piglet is shown in Figure 1. The prominent findings were a rising ICP and, in parallel, a declining $\mathrm{HbO}_{2}$ and $\mathrm{HbD}$. In all experiments, CPP (derived from the difference between MAP and ICP) decreased with increasing ICP (despite a consistent small compensatory increase in MAP with increasing ICP), with the maximal declines to approximately $50 \%$ of baseline values observed at the highest levels of ICP $(40-50 \mathrm{~mm} \mathrm{Hg})$ (data not shown).

Changes in CPP and the resultant changes in $\mathrm{HbO}_{2}, \mathrm{Hb}$, and $\mathrm{HbD}$ for all animals were analyzed for the relationships between the NIRS signals, the microsphere determinations of $\mathrm{rCBF}$, and the CPP. With decreasing CPP, there was a notable decrease in $\mathrm{HbO}_{2}(p=0.001)$, a modest increase in $\mathrm{Hb}(p=$ $0.003)$, and thus a marked decline in $\operatorname{HbD}(p<0.0001)$ (Fig. $2, A-C)$. A nonlinear sigmoidal curve showed a better statistical fit for the relationship between $\triangle \mathrm{HbD}$ and $\triangle \mathrm{CPP}$ than did the linear model, as shown in Figure $2 D(p=0.004$ for the likelihood ratio test). There was a parallel highly significant decrease in $\mathrm{rCBF}$ (as measured by radioactive microspheres) in cerebral cortex, periventricular white matter, and basal ganglia accompanying the decrease in CPP (data not shown). There was no consistent relationship between the changes in $\mathrm{HbT}$
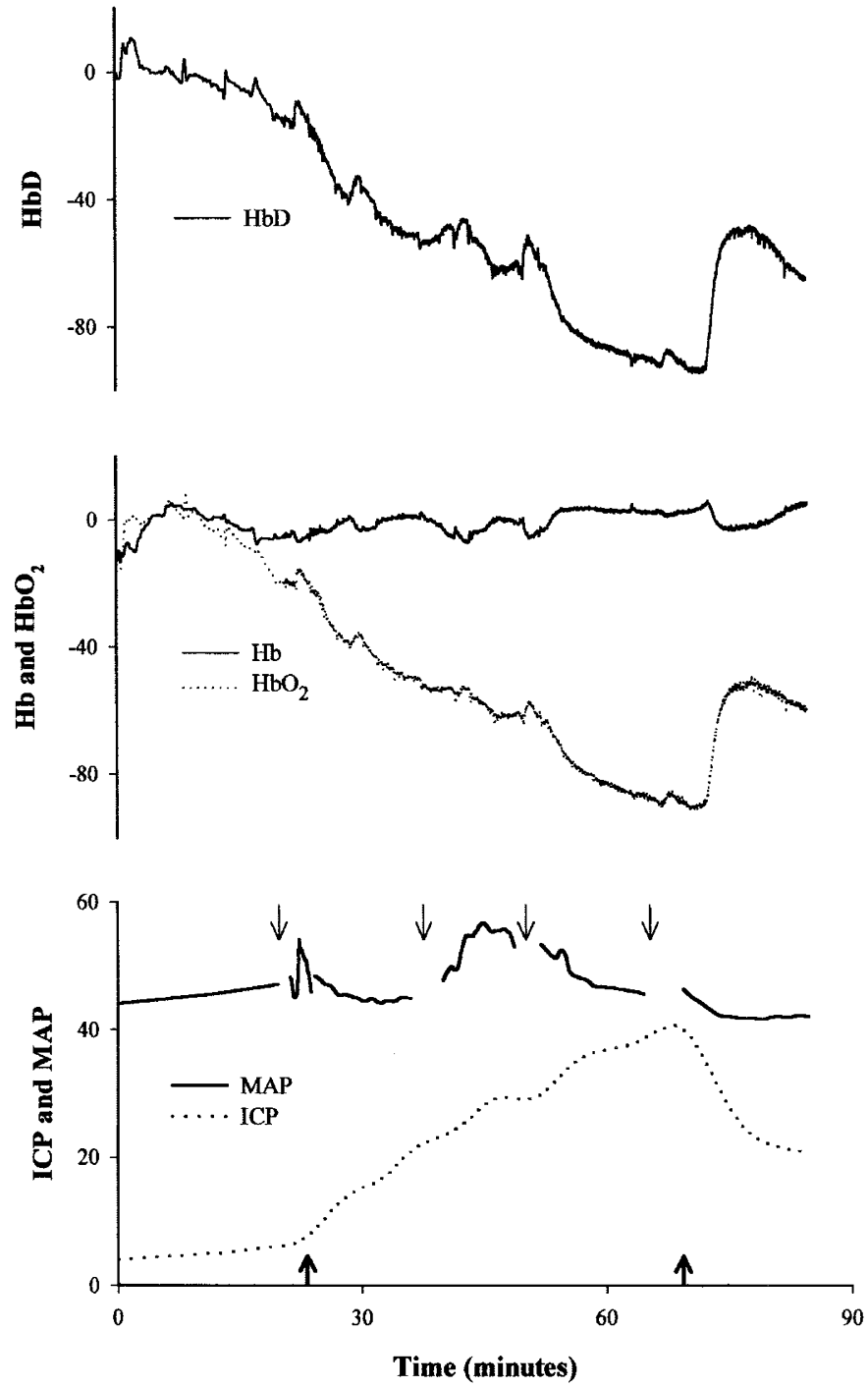

Figure 1. Graphs showing time course of changes in ICP, MAP, $\mathrm{Hb}$, and $\mathrm{HbO}_{2}$ and the resultant change in $\mathrm{HbD}$ during one experiment. Thick arrows on the abscissa indicate start and end of infusion of mock CSF. Thin arrows indicate times when $\mathrm{CBF}$ measurements were made with radioactive microspheres. Interruption of the recording of MAP occurred due to withdrawal of the reference arterial blood sample. ICP and MAP are expressed in $\mathrm{mm} \mathrm{Hg}$ and NIRS signals in $\mu \mathrm{mol} / \mathrm{L} \times \mathrm{dpf}$.

(reflecting cerebral blood volume) or CytOx and the changes in CPP.

Notably, there was a very strong correlation between changes in $\mathrm{HbD}$ and changes in $\mathrm{rCBF}$ in all three brain regions measured. The relationship was highly statistically significant in the cerebral cortex (Fig. $3 A$ ), periventricular white matter (Fig. $3 B$ ), and in the basal ganglia (data not shown) $(p<$ 0.0001 in all three cases).

\section{DISCUSSION}

Data obtained in this study show that in a model of acute hydrocephalus, the decreases in CPP caused by increased ICP result in impaired cerebral perfusion. Importantly, the changes in cerebral perfusion are detected by the noninvasive measurement by NIRS of intravascular Hb signals, particularly HbD. The changes in $\mathrm{HbD}$ correlate tightly with simultaneous mea- 

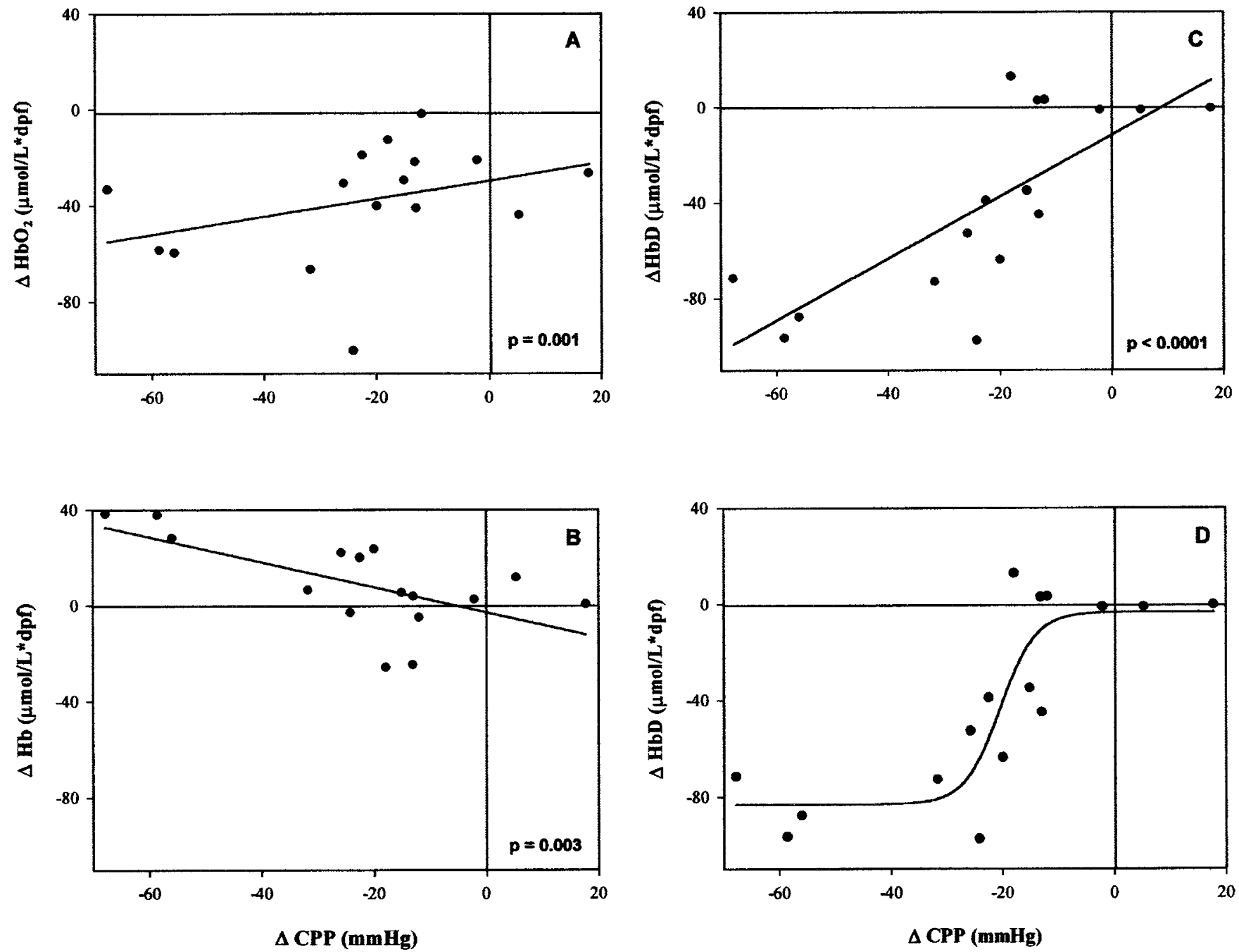

Figure 2. Graphs showing relationship between change in NIRS signals and change in CPP for all animals with accompanying $p$ values. $(A-C)$ Linear regression curves; $(D)$ nonlinear sigmoidal curve for the plot of $\Delta \mathrm{HbD}$ vs $\Delta \mathrm{CPP}$ (compare with $C$ ).

surements of changes in $\mathrm{rCBF}$. The data have implications for the detection of changes in cerebral perfusion in hydrocephalus and for the general value of NIRS, particularly the HbD signal, in the definition of such changes.

This piglet model of acute hydrocephalus proved to be an excellent paradigm for the study of the changes in cerebral hemodynamics caused by the hydrocephalic process. As expected, there was a progressive albeit small increase in MAP with increasing ICP that was attributed to the well-known Cushing response. However, CPP declined with increasing ICP, demonstrating that this mechanism was insufficient to maintain the CPP. Presumably, the decline in CPP was primarily responsible for the observed decreases in $\mathrm{CBF}$ in the three brain regions, as documented by radioactively labeled microspheres. Decreased CBF has been observed in a variety of experimental models of hydrocephalus (2-4).

Our comparisons of the NIRS signals with the rCBF measurements demonstrate the ability of NIRS to detect the observed changes in cerebral hemodynamics. The fall in CBF shown by the microsphere technique is indicative of a decrease in arterial blood flow to the brain due to the observed decline in CPP. Thus, it may be predicted that NIRS would detect an accompanying decline in $\mathrm{HbO}_{2}$ with increasing ICP because changes in $\mathrm{HbO}_{2}$, the dominant hemoglobin chromophore in the well-oxygenated arterial compartment, would be expected to result from changes in arterial flow. In fact, there was a significant correlation between the decrease in CPP and the decrease in $\mathrm{HbO}_{2}$. However, a more accurate measure of cerebral perfusion would be the determination of cerebral intravascular oxygenation and oxygen delivery, which are reflected by the difference between the $\mathrm{HbO}_{2}$ and $\mathrm{Hb}$ signals, i.e. $\mathrm{HbD}$. Indeed, changes in $\mathrm{HbD}$ correlated more strongly with the changes in $\mathrm{rCBF}$ than did $\mathrm{HbO}_{2}$. This situation resulted because $\mathrm{Hb}$ increased modestly as $\mathrm{CPP}$ and $\mathrm{rCBF}$ declined, perhaps because of an increase in oxygen extraction provoked by the decreased cerebral perfusion.

It is interesting to note that the relationship between $\Delta \mathrm{HbD}$ and $\triangle$ CPP was better described by a sigmoidal curve than a linear regression (Fig. 2, $C$ and $D$ ), although this distinction was not part of our original hypothesis. There appeared to be no significant changes in $\mathrm{HbD}$ resulting from the small initial decreases in CPP. However, the subsequent larger declines in $\mathrm{CPP}$ resulted in a significant decrease in $\mathrm{HbD}$ (and CBF). This finding suggests that there may be a critical threshold of CPP 

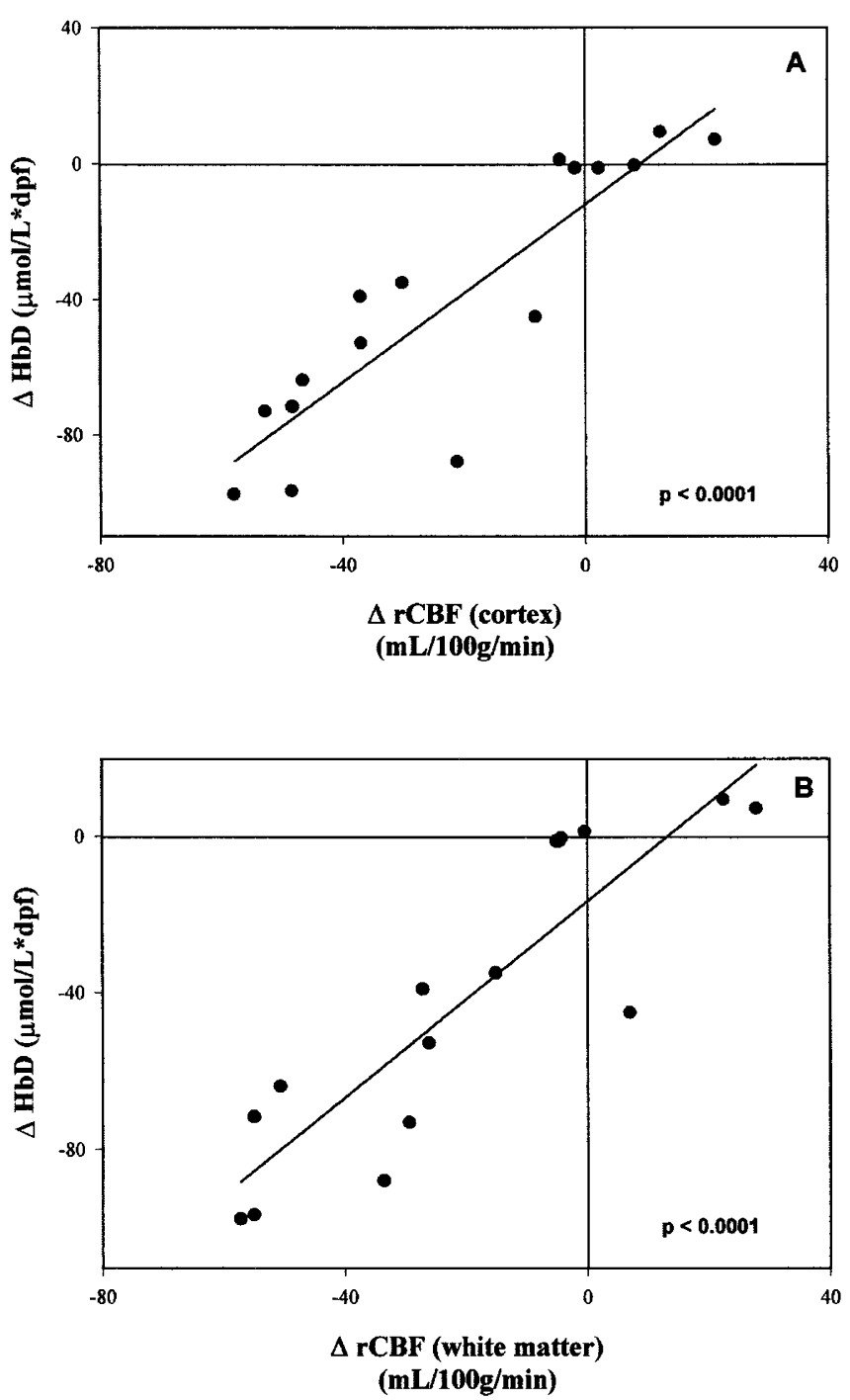

Figure 3. Graphs showing the highly significant relationship between change in $\mathrm{HbD}$ and the change in $\mathrm{rCBF}$ in cerebral cortex $(A)$ and cerebral white matter $(B)$ with accompanying $p$ values.

below which significant impairments in $\mathrm{CBF}$ and cerebral oxygenation occur. This threshold may reflect the limits of cerebrovascular autoregulation. Further declines in $\mathrm{HbD}$ (and, thus, $\mathrm{CBF}$ ) were not observed below very low values of CPP. However, larger studies would be needed to confirm this sigmoidal relationship, as there are only a small number of data points at extremes of the curve.

The demonstration in this piglet model of hydrocephalus of a tightly correlated and highly significant relationship between $\mathrm{HbD}$ and $\mathrm{rCBF}$ in all three brain regions studied is consistent with our previous observation that changes in $\mathrm{HbD}$ reflected changes in CBF in a piglet model of hypotension (11). In that study, an aortic ligature was used to produce an abrupt fall in systemic MAP to $<50 \%$ of baseline MAP and, thereby, an abrupt decrease in CPP. Parallel falls in $\mathrm{HbD}$ and CBF (as measured by NIRS and radioactive microspheres, respectively) were observed. In the current model, the decline in CPP was produced by an increase in ICP rather than a decrease in MAP and, thus, shows that the correlation between $\mathrm{HbD}$ and $\mathrm{rCBF}$ holds true in paradigms of declining $\mathrm{CBF}$ produced by two quite different manipulations.

The potential value of a technique providing the capability to monitor CBF noninvasively and continuously at the bedside in hydrocephalus is substantial. Indeed, this ability would be valuable in the study of a number of pathologic processes involving altered cerebral hemodynamics in the human newborn. The information derived could lead to timely and rational interventions.

Acknowledgments. The authors thank Gene Walter, R. EEG $\mathrm{T}$, and Patricia Dunning, RT(R), for their excellent technical assistance and Shaye Moore for help in manuscript preparation.

\section{REFERENCES}

1. Volpe JJ 1995 Intracranial hemorrhage. In: Neurology of the Newborn. WB Saunders, Philadelphia, pp 373-463

2. Jones HC, Richards HK, Bucknall RM, Pickar JD 1993 Local cerebral blood flow in rats with congenital hydrocephalus. J Cereb Blood Flow Metab 13:531-534

3. Del Bigio M, Bruni J 1988 Changes in periventricular vasculature of rabbit brain following induction of hydrocephalus and after shunting. J Neurosurg 69:115-120

4. da Silva MC, Michowicz S, Drake JM, Chumas PD, Tuor UI 1995 Reduced local cerebral blood flow in periventricular white matter in experimental neonatal hydrocephalus-restoration with CSF shunting. J Cereb Blood Flow Metab 15:1057-1065

5. Hill A, Volpe JJ 1982 Decrease in pulsatile flow in the anterior cerebral artery in infantile hydrocephalus. Pediatrics 69:4-7

6. Lui K, Hellmann J, Sprigg A, Daneman A 1990 Cerebral blood-flow velocity patterns in post-hemorrhagic ventricular dilation. Childs Nerv Syst 6:250-253

7. Chumas P, Drake J, Del Bigio M, da Silva M, Tuor U 1994 Anaerobic glycolysis preceding white-matter destruction in experimental neonatal hydrocephalus. J Neurosurg 80:491-501

8. da Silva M, Drake J, Lemaire C, Cross A, Tuor U 1994 High-energy phosphate metabolism in a neonatal model of hydrocephalus before and after shunting. J Neurosurg 81:544-553

9. Taylor GA, Short B, Walker L, Traystman R 1990 Intracranial blood flow: quantification with duplex Doppler and color Doppler flow US. Radiology 176:231-236

10. Soul JS, du Plessis AJ 1999 New technologies in pediatric neurology. Near-infrared spectroscopy. Semin Pediatr Neurol 6:101-110

11. Tsuji M, duPlessis A, Taylor G, Crocker R, Volpe JJ 1998 Near infrared spectroscopy detects cerebral ischemia during hypotension in piglets. Pediatr Res 44:591-595

12. Wray S, Cope M, Delpy D, Wyatt J, Reynolds E 1988 Characterization of the near infrared absorption spectra of cytochrome aa3 and haemoglobin for the non-invasive monitoring of cerebral oxygenation. Biochim Biophys Acta 933:184-192

13. Wyatt J, Delpy DT, Cope M, Wray S, Reynolds E 1986 Quantification of cerebral oxygenation and hemodynamics in sick newborn infants by near infrared spectroscopy. Lancet 2:1063-1066 